\title{
Urban and Peri-urban Population Dynamics in Case Study Cities within Europe
}

EMMA TERÄMÄ, Dr.Sc. (tech.), Research Scholar

World Population Program, International Institute for Applied Systems Analysis, IIASA, Austria

\begin{abstract}
This article deals with population dynamics in chosen European locations with different age structures, future expected growth developments and urbanization prospects. The case study approach was chosen in order to depict spatially relevant details and characteristics of a small region with an urban core. The populations are subjected to a set of four possible futures, which are called scenarios. The demographics of each are different, and these variations are shown by means of population projections. The results show that in Koper, Slovenia and the Warsaw region, Poland the cities undergo dynamics that is very sensitive to the age structure of the population, future fertility, mortality and international migration prospects. The degree of urbanization further depends on local movement of population, which can be muffled by daily work-migration. Data availability on the local level is proven to be a challenge for detailed demographic studies, as is future uncertainty with respect to migration, be it local or international. Despite the uncertainties in the future levels of migration, it can be seen that age-structure and current low fertility levels have a long-standing impact on the demography of a region.
\end{abstract}

Keywords: aging, energy crisis, IPCC, low fertility, urbanization

\section{Introduction}

Contemporary urbanisation processes are manifold and can be manifested as regional urban development. The phenomenon of urban development has attracted the interest of scholars and planners for over a century, giving rise to numerous urbanisation concepts. An early formulation by Howard (1898) in response to the rapid growth of London, also called 'Social City', was based on and around the city-region, and this concept, with numerous variations, has been a source for urban and regional planners ever since. Subsequent work on the topic includes Cities in Evolution (Geddes 1915), The Metropolitan Community (Mckenzie 1933), The Urban Field (Friedmann \& Miller 1965), The Containment of Urban England (Hall et al. 1973), City Growth Cycles (van den Berg et al. 1982) and the Differential Urbanisation Concept (Geyer and Kontoly 1996). More recent studies focusing on locations in the UK include Sociable Cities (Hall \& Ward 1998) and City Region 2020 (Ravetz 2000). 
The above examples are based on an urban core and its associated commuting ring, which includes peri-urban and rural areas. The peri-urban area is often defined as the area immediately surrounding the man-made urban area, but at the same time containing a mixture of urban and rural activities and land uses (Adell 1999). At the same time it is often an area under urban influence due to commuting, but with simultaneously a rural character due to an agro-forestry sector that represents an important part of the total land surface (Caruso 2005).

The evolution of concepts reflects constantly changing urbanisation trends: the spatial concentration and selective suburbanisation until the 1970s, the counterurbanisation of the 1980s, and the dominance of metropol formation during the 1990s. At present, the latter is assumed to prevail throughout East and West-Europe. It is also often assumed that urban development is not only descriptive of its time but region. The United Nation's World Urbanization Prospects (UN 2006) takes this as a starting point for their general approach assuming fast growing cities in most developing regions ranging from Asia to Africa and Latin America. However, this concept has achieved criticism in the field of urbanization and development studies (Cohen 2004).

The current definitions have, however, their limitations. They have mainly been examined from the point of view of structural properties (e.g. polycentricity) of broader urban systems, and of their economic and social performance. So far, studies have also mainly been following static approaches, leaving outside dynamic phenomena and consequences such as the rapid demographic change in Europe, namely population ageing and migration.

In this study I will perform a detailed analysis of the dynamics between the rural-periurban and urban zones within the case studies including a future-oriented perspective where responses to global drivers will be included according to scenarios developed for this purpose. The aim is to investigate how dependant on different demographic drivers the future development of cities is. Can urbanization be related to specific demographic trends already visible, or will it all boil down to migration and the uncertainties that brings? It is also interesting to compare cities' development inside a region as presumably uniform as Europe is.

The scenarios explicitly include demographic drivers changing according to different 'shock' assumptions about the future. These were developed based on the wellknown IPCC scenarios (Nakićenović and Swart 2000) that in addition to the energy and economic assumptions about the future, also contain a number of imbedded demographic drivers. However, in order to accentuate future uncertainty and possible variation in outcomes of different scenarios, it was desired to create more extreme cases than the 'regular' IPCC scenarios. Therefore the shocks administered were based on the following four alternate futures: 1) Hyper-tech, i.e. highly technocratic society with telecommuting work-possibilities and wealthy societies and stable economic 
growth. 2) Water-crisis in all forms, i.e. draughts in the South combined with flooding in the North and dramatic sea level rise. 3) Carbon sensitive future, with emphasis on renewable energy source utilization and diminished energy expenditures in both private and corporate sectors. 4) Social fragmentation, which is the last of the four scenarios, and depicts a future with increased social inequality. This can arise through a segregated society in terms of nationality, country of origin, age etc. It is envisioned that the fourth scenario would be a so-called 'failed-EU' scenario where the ideals of uniform legislation (constitution etc.) do not withhold. None of the provided scenarios are so-called business as usual-scenarios.

\section{Case Studies}

The first of the cases considered here is the city of Koper, the only harbor city in Slovenia with under 50000 inhabitants. It is an industrial urban center that is nevertheless dependant on tourism, and experiences high levels of daily work migration. It is expected to grow in terms of the urban population. Low fertility and rising life expectancy may, however, play a crucial role in determining the future size of Koper that has not experienced great international nor internal levels of migration.

Warsaw is considered as part of a larger area, namely the NUTS2-size voivodship of Mazowieckie. At the beginning of our study in 2005 there are a total of 5.1 million inhabitants in Mazowieckie of which some 1.8 million live in rural areas and the rest in urban zones. In 2003 the population of Warsaw alone was almost 1.7 million (Statistical Office in Warsaw, 2005). This means that at the beginning of this study, all the other urban areas in the voivodship combined, contained less people than Warsaw. This big metropol of the East has a steady stream of in- and out-migration, relatively low levels of fertility and a particularly fast aging population. The city is developing an increasing gender imbalance due to the life expectancy of women overweighing that of the men by several years. This is also shown by the population pyramids that depict the levels of men and women in this capital city and surround area of Poland.

\section{Data and Methods}

The case study areas were chosen in an EU-consortium project called PLUREL, where peri-urban land use change considerations on different scales are addressed. A report on demographic methodology on various scales has been made public previously (Skirbekk et al. 2007). In that paper, we describe the use of population projections for estimating future population sizes and structure on various scales from national to subnational, and local levels as applied here. The project PLUREL describes a set of four scenarios for alternative future development that also encompass demographic details (Ravetz 2008). We implement these scenarios in our city case studies, and complete all population projections in line with these four alternate futures. 
Population projections conducted on smaller scales can be a useful way of entering detailed information into population forecasting that is lacking on higher levels with more aggregation. The movement of people into and out of cities can only be captured by means of explicit input into population projection models. In this study, the traditional cohort-component method was used in a multi-state application ${ }^{1}$ that enables the simultaneous projection of several population segments. Here these states were chosen according to type of dwelling: urban, peri-urban or rural. The model enables movement of the population from one state to the other. This is a special case of a component method, which is defined simply by the use of estimates or projections of births, deaths, and net migration to update a population (Hollmann et al. 2000).

Population estimates for the future are based on current statistics and alternative interpretations of ongoing trends for the future. These assumptions are often based on expert judgement (Lutz et al. 2000), and/or made consistent to other reliable sources, such as the European statistical office and the U.S. Census Bureau that have a longstanding reputation for carrying out national and international population projections on several levels.

Medium, high and low fertility assumptions used for the fertility analysis in this paper are consistent with Eurostat ${ }^{2}$ assumptions for the future. Detailed population, migration and mortality information was obtained from the national statistical offices, or regional city offices where applicable.

The detailed scenario assumptions including demographic indicators for the population projections are summarized below. The scenarios are based on the IPCC report (Nakićenović and Swart 2000), with added 'shock' effects. This means, that there is no baseline or 'middle of the road' scenario, nor a scenario that would be considered the most likely future. Instead, every one of the below scenarios is a unique combination of carefully considered changes in the indicators rendering a picture of a possible 'shock' future from the population perspective.

The 'Tech' technological advances - shock is based on the A1 IPCC scenario. Respectively 'Water' is A2 with a water crisis shock scenario, 'Carbon' depicts the reduced carbon emissions future relating to IPCC B1, and 'Social' is short for the socially fragmented future scenario, or B2 in IPCC terminology. A more elaborate description of the underlying scenarios has been documented previously (Ravetz 2008).

\footnotetext{
${ }^{1} \mathrm{http} / / / \mathrm{www}$. iiasa.ac.at/Research/POP/pub/software.html?sb=25

${ }^{2} \mathrm{http} / / /$ epp.eurostat.ec.europa.eu
} 
Table 1. Scenario assumptions.

\begin{tabular}{|l|c|c|c|c|c|}
\hline & Pop & Fertility & Mortality & $\begin{array}{c}\text { International } \\
\text { migration }\end{array}$ & Urbanization \\
\hline A1 Tech & Med-high & Med & Low & Med & Low \\
\hline A2 Water & Med & Med & Med & Med & High \\
\hline B1 Carbon & Low & Low & High & Low & High \\
\hline B2 Social & Med & Med & Med & Med & Low \\
\hline
\end{tabular}

The level of the given indicators, namely medium, high or low is linked, where possible, to other sources of assumptions for demography. Therefore as basis for the fertility assumptions are used the Eurostat estimated of low or medium fertility levels on the national level. The Eurostat one-year age group assumptions were aggregated to five-year age specific fertility rates (ASFRs) and the period ASFRs closest to our last projection period were used. Interpolation was carried out between the first and the last projection periods to enable a smooth transition from the current fertility levels to the final projection period.

Low mortality is considered lower than the current experienced mortality rates, with the current rates being provided by the regional statistical offices. High rates are higher than the ones currently observed, meaning a turn-around of the long experienced increasing life expectancy. This is applied to the B1 'carbon sensitive' scenario with an overall low population expectation. For low mortality the factor 0.75 was used for the last projection period and interpolation carried out between the first $(1.0=$ current $)$ and the last period in order to obtain a smooth transition. Together, low mortality coupled to medium fertility gives a medium to high future population estimate as in the A1 scenario, whereas high mortality and low fertility provide the low population estimate as can be seen in the case of B1. The remaining two scenarios follow a medium trend in population development nevertheless with different assumptions in the degree of urbanization.

International migration was not particularly addressed in this study but came in the form of net migration as obtained from the statistical offices. This is due to the nature of the chosen shock scenarios: they were assumed to affect the whole globe much in the way that the current economic crisis does, and thus giving little incentive for a great change in international migration from the current levels. Also the fact that international conflicts on the large scale are impossible to forecast, and this study being a regional study it was further not feasible to assume that any of the small areas depicted here would get a major share of, say, international migration due to people displaced by wars.

The urbanization and local movement of population was estimated for the future as a multiple of the current trend, either emphasizing or mellowing the status quo. In cases where no age structure was given in the data available, the Rogers and Castro model for migration schedules was used (Rogers 1984). 


\section{Results and Discussion}

\section{Koper}

Population projections were carried out in the case of Koper for the time frame 2006 to 2026 and for Warsaw region from 2005 to 2030. Fig. 1 shows what the population pyramid is like in 2006, the projection starting year for Koper, therefore giving a reference point for the discussion on the effects of the scenarios. For the end point, 2026, two examples of population pyramids are shown. Figures 2 and 3 show the development of the population for the two chosen scenarios, namely A1 (Hyper-tech) and B1 (Carbon sensitive). Comparing the pyramids one can graphically see the effect of the changed demographic assumptions for the future.

The Koper results on population development show a marked increase in settlement in the peri-urban areas for scenario A1. A calculation on population dynamics is carried out for the end population of the four scenarios, seeking to show the direction of the population movement. From Table 2 it becomes clear that the low urbanization assumptions in scenarios A1 and B2 are correctly depicted in the results. Those are the two scenarios where the urban population steadily decreases with time. It was against prior expectations that we find the core urban population in Koper to be decreasing throughout the scenarios (see Table 2). Even for the more positive urbanization scenarios the urban population decreases until the very end of the projection period. Looking at the ratio of urban population to the combined peri-urban and rural population the situation does not change much. A partial interpretation for this could be the increased well-being in the society that facilitates work-migration and choice of where to live, even if further away from the cities.

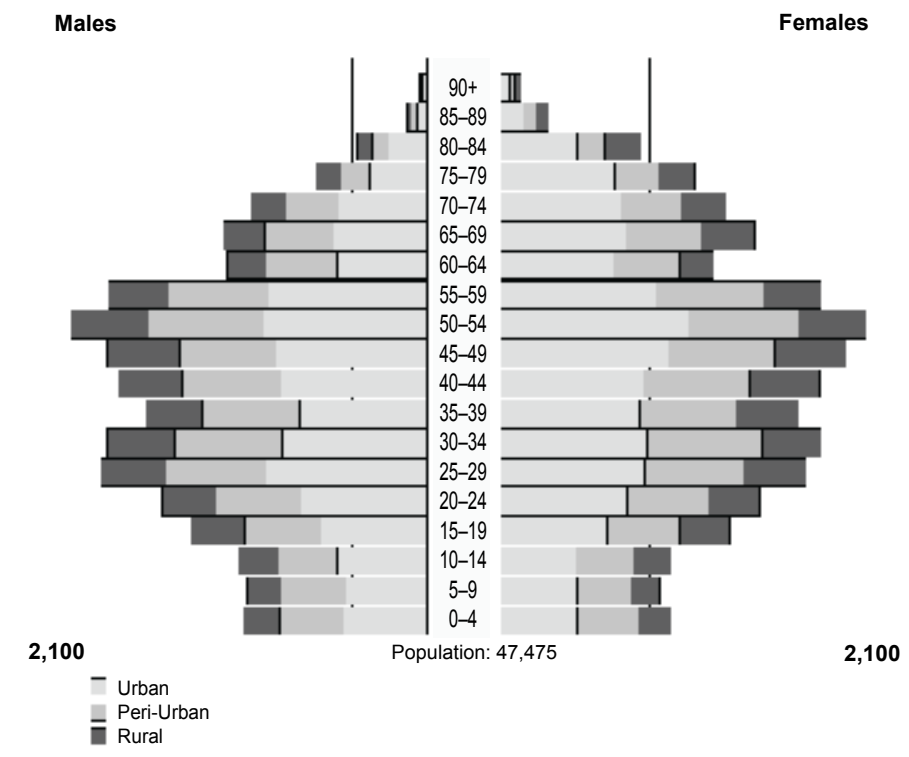

Figure 1. Population Projection of Koper-Slovenia 2006 
The appendix shows in tabular form the population of Koper broken down by area, year and sex for the four different scenarios.

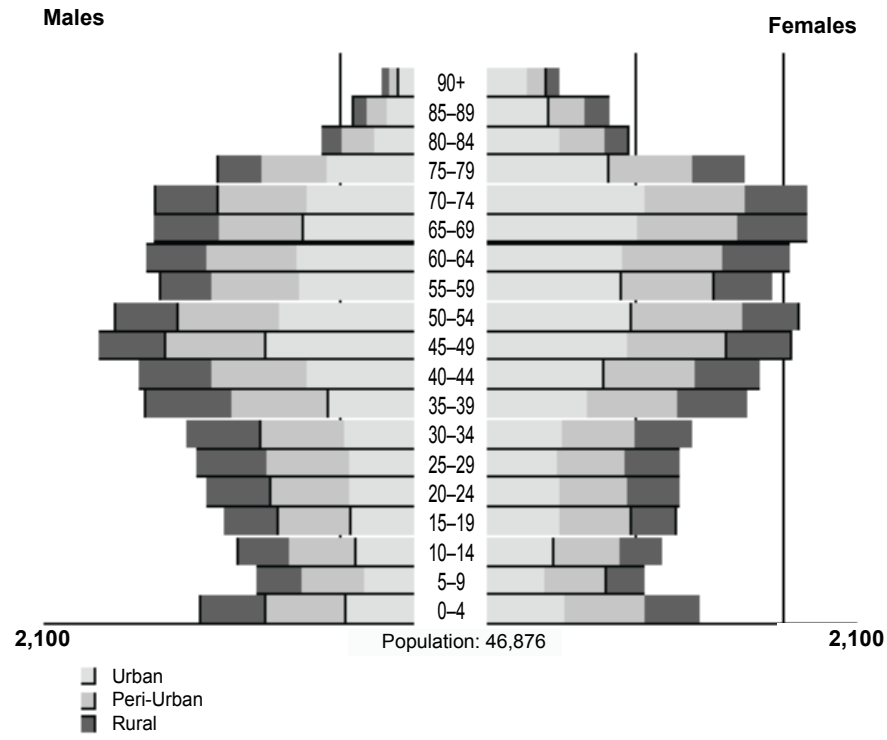

Figure 2. Population Projection of Koper-Slovenia 2026.

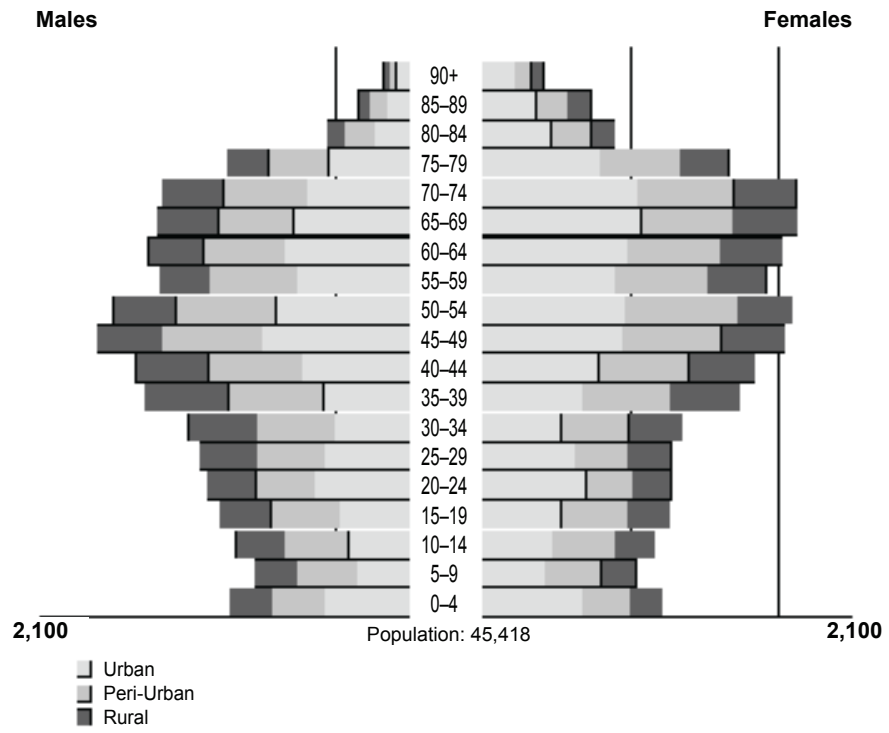

Figure 3. Population projection of Koper-Slovenia 2026, variant B1. 
Table 2. Population ratios showing the dynamics of the population change.

\begin{tabular}{ccccccccc}
\hline & \multicolumn{2}{c}{ A1 } & \multicolumn{2}{c}{ A2 } & \multicolumn{2}{c}{ B1 } & \multicolumn{2}{c}{ B2 } \\
& U/P-U & U/(P-U+R) & U/P-U & U/(P-U+R) & U/P-U & U/(P-U+R) & U/P-U & U/(P-U+R) \\
\hline $\mathbf{2 0 0 6}$ & 1.52 & 0.923 & 1.52 & 0.924 & 1.52 & 0.923 & 1.52 & 0.923 \\
$\mathbf{2 0 1 1}$ & 1.41 & 0.852 & 1.41 & 0.852 & 1.41 & 0.852 & 1.41 & 0.852 \\
$\mathbf{2 0 1 6}$ & 1.33 & 0.798 & 1.39 & 0.829 & 1.39 & 0.832 & 1.33 & 0.793 \\
$\mathbf{2 0 2 1}$ & 1.27 & 0.749 & 1.41 & 0.836 & 1.42 & 0.840 & 1.25 & 0.738 \\
$\mathbf{2 0 2 6}$ & 1.19 & 0.699 & 1.50 & 0.876 & 1.50 & 0.881 & 1.18 & 0.689 \\
\hline
\end{tabular}

Key: U - urban, P-U - peri-urban, R - rural population.

\section{Warsaw}

The second projected region was Warsaw and its surroundings. From the Warsaw population pyramids one result is evident. There is a persistent gender bias towards more women. This is true for all the scenarios alike. Figure 4 shows the starting year 2005 population of Warsaw region (voivodship of Mazowieckie). The two scenarios whose final projection periods can be compared in Figures 5 and 7 are those of A1 and A2. The A2 (water crisis) was dealt with a particularly high urbanization assumption that created the visible rise in urban population as seen in Figure 8. All other scenarios, including the moderate version of A2, ended up in a decreasing overall, as well as urban population, despite of the urbanization favouring input of localized population movement in A2 and B1. The urban areas of Mazowieckie are thus expected to experience population decline together with a high degree of elderly women. This despite of the fact that the life expectancy for women was allowed to increase (in A1 with low mortality) only moderately, from 79 to 82 years whereas that of the men increased in the same period from below 71 to over 75 . The especially low birth rates were expected to remain so in the scenario B1. In A1 (hyper-tech) the total fertility rate was allowed to increase from 1.25 to a moderate 1.29 as can be expected for an economic growth and well-being scenario.

The results of this study indicate that projections can be a useful tool in estimating both possible, and under certain circumstances, probable population futures. It is found, that without explicit and region specific demographically detailed data it is impossible to create an understanding of the likelihood or prospects of the future of urbanization in a region. National level data may capture foreign migration levels more accurately, however they do not give particulars of where the people are migrating inside a country. A functioning work-force migration, i.e. permanent residence outside of town may be enough to offset in cities a rather large international in-migration. Therefore more studies are needed on the rural-urban level that correctly present where the local population prefers to live, in order to make more accurate assumptions about future urbanization levels. This study shows, that in the time of increasing life expectancy and low, even lowest low fertility levels, decreasing urban populations are still more likely in the next 20-30 years due to the large cohorts (born in the 40s and 50s) slowly deceasing. 


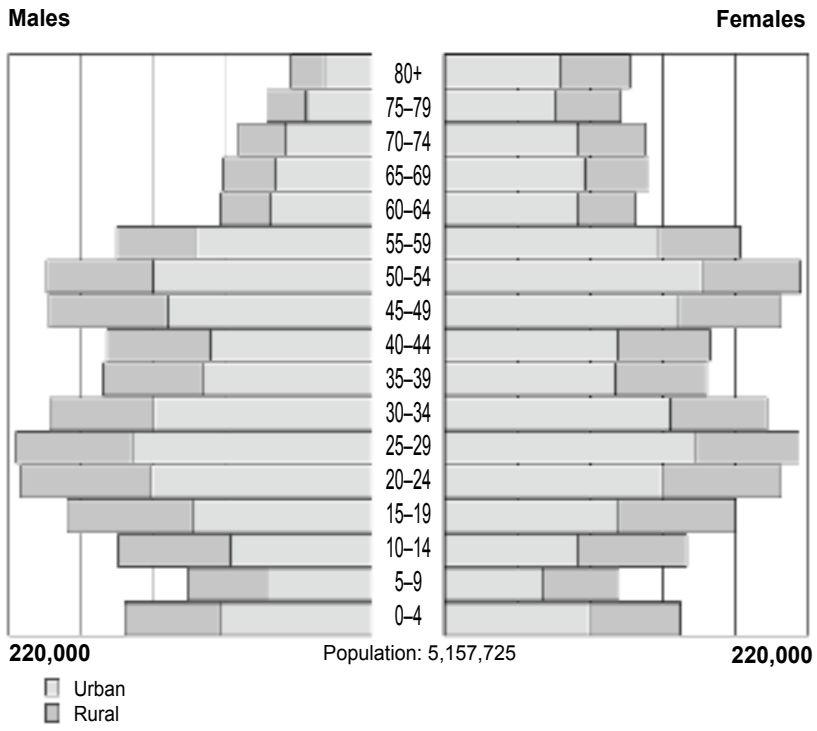

Figure 4. Population projection of Warsaw region 2005.

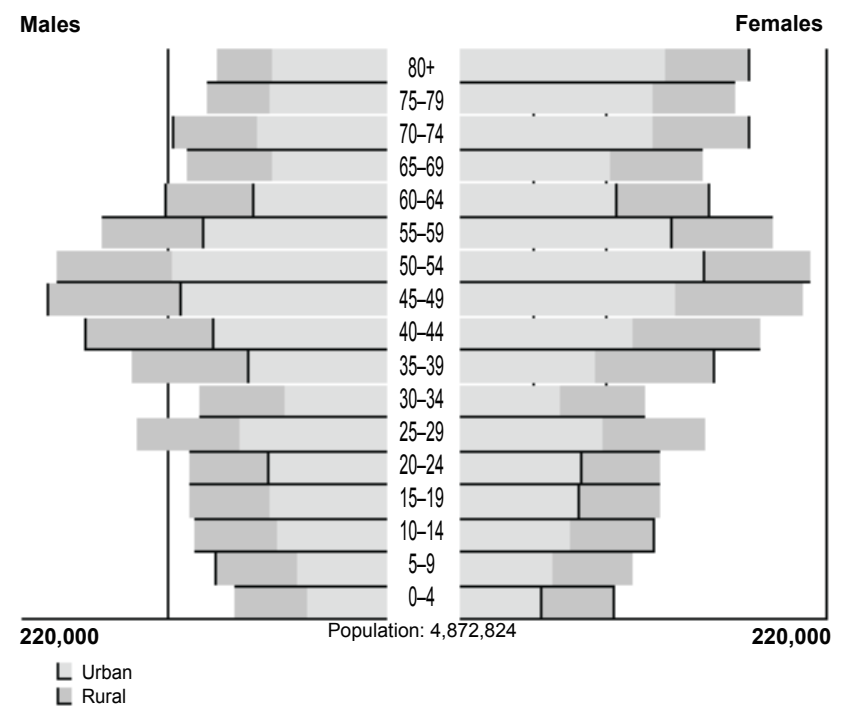

Figure 5. Population projection of Warsaw region 2030.

Therefore the age structure that is currently visible guides us demographers well, as long as the initial data is detailed enough in all the relevant aspects, namely fertility, mortality and migration. Further, in the present study the time span of about 20 years into the future does not provide enough variation in terms of different outcomes due to changes in assumptions of future fertility and mortality levels. For a more detailed study on the effect of e.g. extreme high life expectancy or continuing lowest low fertility 
118 Urban and Peri-urban Population Dynamics in Case Study Cities

levels, a longer projection period would be needed. For this study the input of current trends in international and internal migration with small variations for the future, as well as moderate assumptions on fertility and mortality were in good balance for the projection period used.

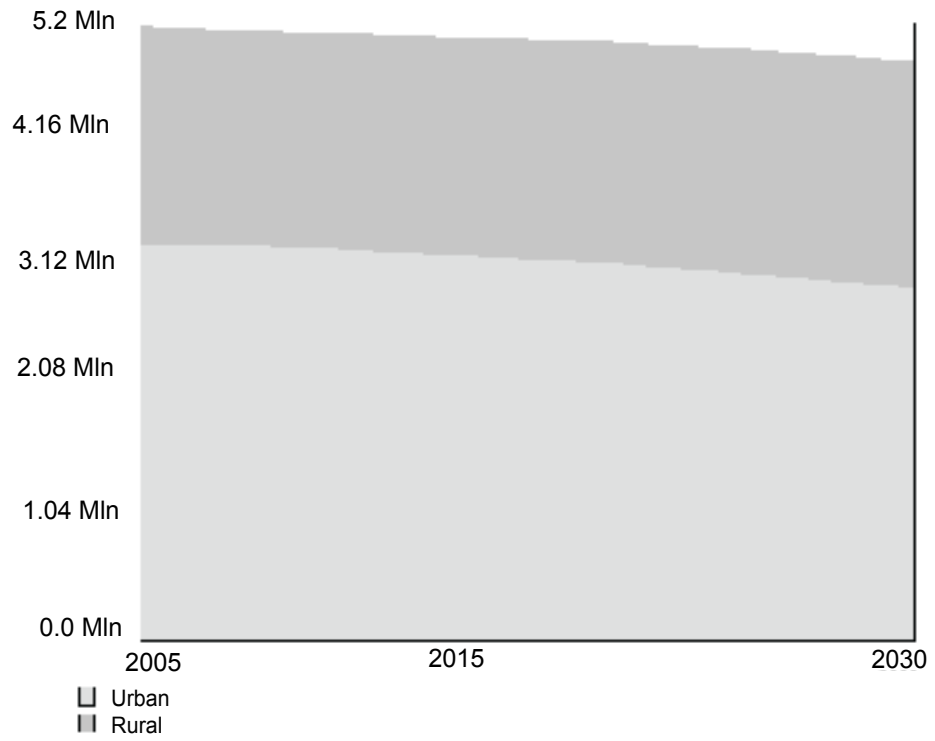

Figure 6. Population projection of Warsaw region, A1

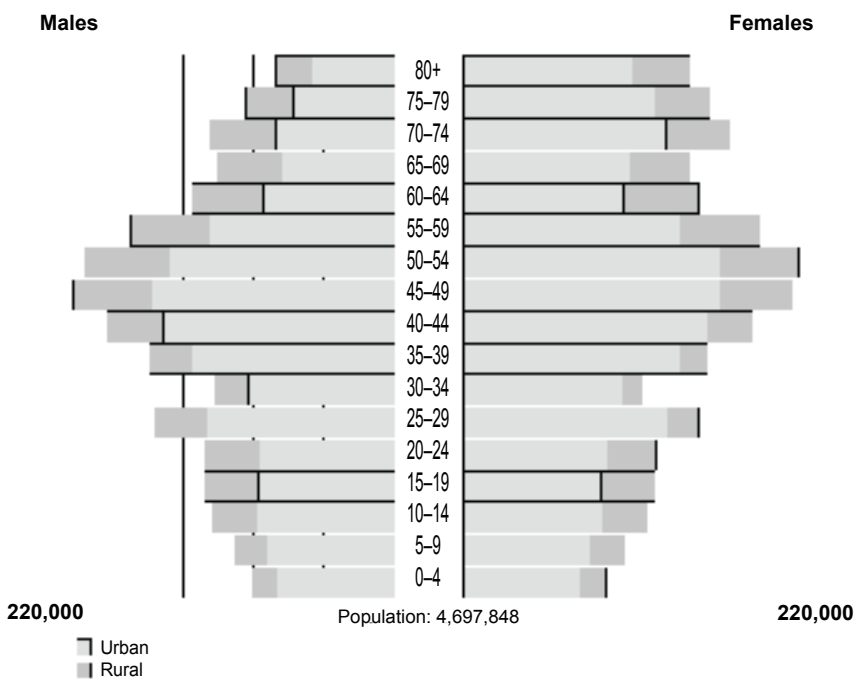

Figure 7.Population Projection of Warsaw Region 2030, A2-urban. 


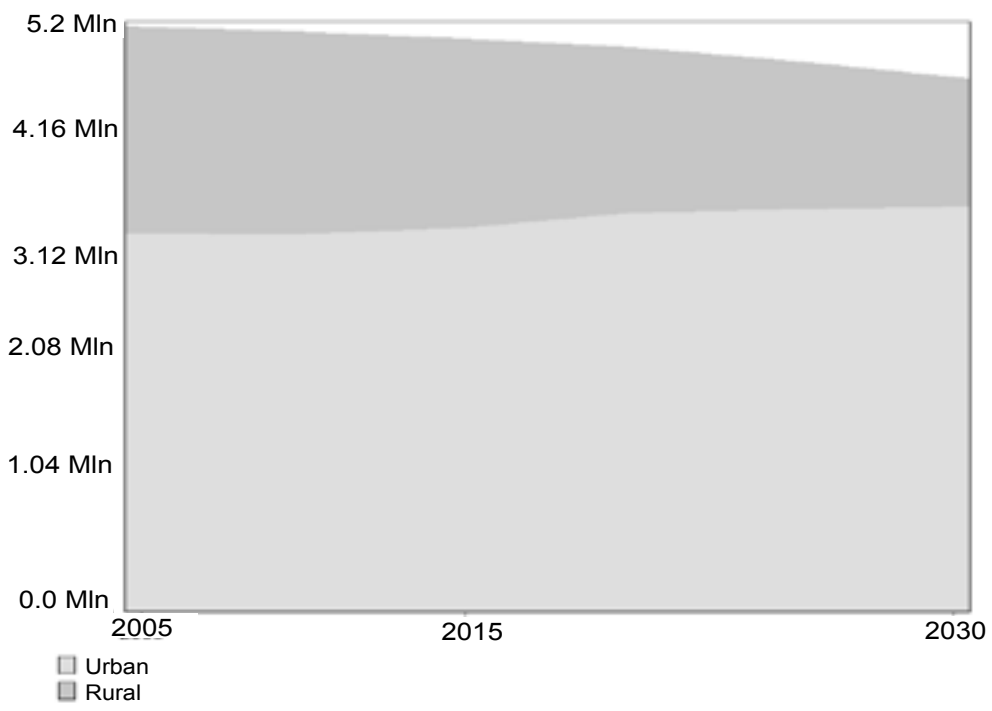

Figure 8. Population Projection of Warsaw Region A2-urban.

\section{Summary and outlook}

We found that in the future population development of cities, many factors are in play. In the work above the demographic indices fertility, mortality and migration were given different assumptions in line with four pre-defined scenarios for the future. Results show that varying these factors even slightly does have an effect, and that the starting population structure has an equally great impact on the future of the population.

In the case study of Koper, the expected strong urbanization trend was not seen. Instead the urban population declined in all scenarios in the beginning, and turned around only slightly in two of the four scenarios. For Warsaw area the aging of the population was extreme. A high urbanization scenario played out to favor urban living in the future but even moderate increase in fertility (that is very low) could not turn around the overall trend of decreasing population size.

Case studies of Leipzig, Germany; Manchester, UK and the Hague, Netherlands have also been completed. These will be described in another context.

\section{Acknowledgments}

The author would like to thank the Academy of Finland for funding and Dr. Erling Lundevaller and Ilkka Keppo for useful comments and discussion. 


\section{References}

Adell, G. 1999. Theories and models of the peri-urban interface: a changing conceptual landscape, Literature review, Strategic environmental planning and management for the peri-urban interface. Research project (SCOPE, PUECH). Development planning unit, University College London, London.

Caruso, G., M.D.A. Rounsevell and G. Cojocaru. 2005. Exploring a spatio-dynamic neighbourhood-based model of residential behaviour in the Brussels periurban area. International Journal of Geographical Information Science 19(2):103-123.

Cohen, Barney. 2004. Urban Growth in Developing Countries: A Review of Current Trends and a Caution Regarding Existing Forecasts. World Development 32(1):23-51.

Friedmann, J. and J. Miller. 1965. The Urban Field. Journal of the American Institute of Planners 31:312-329.

Geddes, P. 1915. Cities in Evolution. An Introduction to the Town Planning Movement and to the Study of Cities. 1968 edition. London: Benn.

Geyer, H.S. and T.M. Kontuly (Eds.). 1996. Differential Urbanisation: Integrating Spatial Models. Arnold. London.

Hall, P., R. Thomas, H. Gracey and R. Drewett. 1973. The Containment of Urban England. 2 volumes. George Allen and Unwin, London.

Hall, P.G. and C. Ward. 1998. Sociable Cities: The legacy of Ebenezer Howard. John Wiley \& Sons, New York etc.

Hollmann, Frederick W., Tammany J. Mulder and Jeffrey E. Kallan. 2000. Population Division Working Paper No. 38, U.S. Census Bureau. Available at http://www. census.gov/population/www/documentation/twps0038/twps0038.html Last visited January 27, 2009.

Howard, E. 1898. To-morrow: A Peaceful Path to Real Reform. Reprinted by Routledge, London.

Lutz, Wolfgang, Pertti Saariluoma, Warren Sanderson and Sergei Scherbov. 2000. New Developments in the Methodology of Expert-and Argument-Based Probabilistic Population Forecasting, IR-00-020, IIASA. Available at http://www.iiasa.ac.at/ Publications/Documents/IR-00-020.pdf Last visited: January 27, 2009.

McKenzie, R.D. 1933. The Metropolitan Community. New York: McGraw-Hill.

Nakićenović, N. and R. Swart (Eds.). 2000. Special Report on Emissions Scenarios. Intergovernmental Panel on Climate Change, Cambridge University Press, Cambridge, p. 612.

Ravetz, Joe. 2000. City-Region 2020: integrated planning for a sustainable environment. Earthscan, with the Town \& Country Planning Association, London.

Ravetz, Joe. 2008. Scenario Framework, PLUREL report D1.3.2.

Rogers, Andrei. 1984. Migration, Urbanization, and Spatial Population Dynamics. Boulder: Westview Press.

Skirbekk, Vegard, Isolde Prommer, Samir K.C., Emma Terama, Emma and Chris Wilson. 2007. Report on methods of demographic projection at multiple levels of aggregation, PLUREL report D1.2.1.

Statistical Office in Warsaw. 2005. Panorama of districts of Warsaw in 2003. Information and statistical papers. Warsaw: Statistical Office. 
United Nations. 2006. World Urbanization Prospects: The 2005 Revision. Department of Economic and Social Affairs, Population Division. CD-R Ed.

Van den Berg, L., R. Drewett, L.H. Klaassen, A. Rossi and C.H.T. Vijverberg. 1982. Urban Europe: a study of growth and decline. Oxford: Pergamon.

\section{Appendix}

A. Table 1. Population of Koper by area according to scenario A1.

\begin{tabular}{llrrr}
\hline \multirow{2}{*}{2006} & Men & Women & Both \\
& Urban & 10,827 & 11,967 & 22,794 \\
& Peri-urban & 7,369 & 7,660 & 15,029 \\
& Rural & 4,724 & 4,928 & 9,652 \\
\hline \multirow{2}{*}{2011} & Urban & 10,281 & 11,649 & 21,930 \\
& Peri-urban & 7,602 & 7,904 & 15,506 \\
& Rural & 5,092 & 5,131 & 10,223 \\
\hline \multirow{2}{*}{$\mathbf{2 0 1 6}$} & Urban & 9,779 & 11,312 & 21,091 \\
& Peri-urban & 7,751 & 8,058 & 15,809 \\
& Rural & 5,362 & 5,271 & 10,633 \\
\hline \multirow{2}{*}{$\mathbf{2 0 2 1}$} & Urban & 9,282 & 10,897 & 20,179 \\
& Peri-urban & 7,816 & 8,134 & 15,950 \\
& Rural & 5,589 & 5,387 & 10,976 \\
\hline & Urban & 8,869 & 10,582 & 19,451 \\
& Peri-urban & 7,987 & 8,306 & 16,293 \\
& Rural & 5,924 & 5,600 & 11,524 \\
\hline
\end{tabular}

A. Table 2. Population of Koper by area according to scenario A2.

\begin{tabular}{llrrr}
\hline & & Men & Women & Both \\
\hline \multirow{2}{*}{2006} & Urban & 10,827 & 11,967 & 22,794 \\
& Peri-urban & 7,369 & 7,660 & 15,029 \\
& Rural & 4,724 & 4,928 & 9,652 \\
\hline \multirow{2}{*}{2011} & Urban & 10,281 & 11,649 & 21,930 \\
& Peri-urban & 7,602 & 7,904 & 15,506 \\
& Rural & 5,092 & 5,131 & 10,223 \\
\hline \multirow{2}{*}{$\mathbf{2 0 1 6}$} & Urban & 10,014 & 11,591 & 21,605 \\
& Peri-urban & 7,649 & 7,930 & 15,579 \\
& Rural & 5,295 & 5,193 & 10,488 \\
\hline \multirow{2}{*}{$\mathbf{2 0 2 6}$} & Urban & 9,939 & 11,683 & 21,622 \\
& Peri-urban & 7,524 & 7,787 & 15,311 \\
& Rural & 5,397 & 5,165 & 10,562 \\
\hline & Urban & 10,036 & 11,988 & 22,024 \\
& Peri-urban & 7,252 & 7,469 & 14,721 \\
& Rural & 5,384 & 5,039 & 10,423 \\
\hline
\end{tabular}


122 Urban and Peri-urban Population Dynamics in Case Study Cities

A. Table 3. Population of Koper by area according to scenario B1.

\begin{tabular}{llrrr}
\hline & & Men & Women & Both \\
\hline \multirow{2}{*}{2006} & Urban & 10,827 & 11,967 & 22,794 \\
& Peri-urban & 7,369 & 7,660 & 15,029 \\
& Rural & 4,724 & 4,928 & 9,652 \\
\hline \multirow{2}{*}{2011} & Urban & 10,281 & 11,649 & 21,930 \\
& Peri-urban & 7,602 & 7,904 & 15,506 \\
& Rural & 5,092 & 5,131 & 10,223 \\
& Urban & 9,983 & 11,557 & 21,540 \\
& Peri-urban & 7,594 & 7,886 & 15,480 \\
& Rural & 5,254 & 5,156 & 10,410 \\
\hline \multirow{2}{*}{2021} & Urban & 9,839 & 11,572 & 21,411 \\
& Peri-urban & 7,402 & 7,686 & 15,088 \\
& Rural & 5,302 & 5,086 & 10,388 \\
\hline \multirow{2}{*}{2026} & Urban & 9,801 & 11,732 & 21,533 \\
& Peri-urban & 7,034 & 7,290 & 14,324 \\
& Rural & 5,212 & 4,902 & 10,114 \\
\hline
\end{tabular}

A. Table 4. Population of Koper by area according to scenario B2.

\begin{tabular}{llrrr}
\hline \multirow{2}{*}{2006} & & Men & Women & \multicolumn{1}{c}{ Both } \\
\hline \multirow{2}{*}{2011} & Urban & 10,827 & 11,967 & 22,794 \\
& Peri-urban & 7,369 & 7,660 & 15,029 \\
& Rural & 4,724 & 4,928 & 9,652 \\
& Urban & 10,281 & 11,649 & 21,930 \\
& Peri-urban & 7,602 & 7,904 & 15,506 \\
& Rural & 5,092 & 5,131 & 10,223 \\
\hline \multirow{2}{*}{2016} & Urban & 9,777 & 11,311 & 21,088 \\
& Peri-urban & 7,783 & 8102 & 15885 \\
& Rural & 5,398 & 5,300 & 10,698 \\
\hline \multirow{2}{*}{2021} & Urban & 9267 & 10,906 & 20,173 \\
& Peri-urban & 7,900 & 8,258 & 16,158 \\
& Rural & 5,693 & 5,472 & 11,165 \\
\hline \multirow{2}{*}{2026} & Urban & 8,741 & 10,493 & 19,234 \\
& Peri-urban & 7,971 & 8,364 & 16,335 \\
& Rural & 5,962 & 5,639 & 11,601 \\
\hline
\end{tabular}

